\title{
THE BIHARMONICITY OF SECTIONS OF THE TANGENT BUNDLE
}

\author{
M. MARKELLOS AND H. URAKAWA
}

\begin{abstract}
The bienergy of a vector field on a Riemannian manifold $(M, g)$ is defined to be the bienergy of the corresponding map $(M, g) \mapsto\left(T M, g_{S}\right)$, where the tangent bundle $T M$ is equipped with the Sasaki metric $g_{S}$. The constrained variational problem is studied, where variations are confined to vector fields, and the corresponding critical point condition characterizes biharmonic vector fields. Furthermore, we prove that if $(M, g)$ is a compact oriented $m$-dimensional Riemannian manifold and $X$ a tangent vector of $M$, then $X$ is a biharmonic vector field of $(M, g)$ if and only if $X$ is parallel. Finally, we give examples of non-parallel biharmonic vector fields in the case which the base manifold $(M, g)$ is non-compact.
\end{abstract}

\section{INTRODUCTION}

The energy functional of a map $\varphi:(M, g) \mapsto(N, h)$ between Riemannian manifolds has been widely investigated by several researchers ([2], [7], [16]) and is given by

$$
E_{1}(\varphi)=\frac{1}{2} \int_{M}\|d \varphi\|^{2} v_{g}
$$

where $d \varphi$ denotes the differential of the map $\varphi$. Critical points for the energy functional are called harmonic maps and have been characterized by the vanishing of the tension field $\tau_{1}(\varphi)=\operatorname{Tr} \nabla d \varphi$.

Let $(M, g)$ be a Riemannian manifold and denote by $\left(T M, g_{S}\right)$ its tangent bundle equipped with the Sasaki metric $g_{S}$. A vector field $X$ on $M$ determines a mapping from $(M, g)$ into $\left(T M, g_{S}\right)$, embedding $M$ into its tangent bundle. If $M$ is compact and orientable, the energy of $X$ is the energy of the corresponding map ([6, pp. 41-42]). Nouhaud ([14]) proved that if $M$ is compact, then $X:(M, g) \mapsto\left(T M, g_{S}\right)$ is an harmonic map if and only if $X$ is parallel. The same conclusion is obtained in [11] but, in this case, the explicit expression of the tension field of the vector field is provided (see subsection 2.2). Gil - Medrano

2000 Mathematics Subject Classification. Primary 58E20; Secondary 53C20.

Key words and phrases. tangent bundle, Sasaki metric, biharmonic maps, critical points. 
([8]) proved that if $M$ is compact, then a vector field $X$ is a critical point of the energy functional $E_{1}$ restricted to the set $\mathfrak{X}(M)$ of all vector fields of $(M, g)$ (equivalently, $X$ is a harmonic vector field) if and only if $X$ is parallel. As a consequence, the search for critical points for the energy functional $E_{1}: C^{\infty}(M, T M) \mapsto[0,+\infty)$ or $E_{1}: \mathfrak{X}(M) \mapsto[0,+\infty)$ shows that both domain $C^{\infty}(M, T M)$ and $\mathfrak{X}(M)$ are inappropriate.

In [7], J.Eells and J.H.Sampson suggested the idea of studying $k$ harmonic maps. A particular interest has the case $k=2$. They define the bienergy of $\varphi$ as the functional

$$
E_{2}(\varphi)=\frac{1}{2} \int_{M}\left\|\tau_{1}(\varphi)\right\|^{2} v_{g}
$$

and a map is biharmonic if and only if it is a critical point of $E_{2}$. Jiang ([12]) derived the associated Euler-Lagrange equation for $E_{2}$. A harmonic map is automatically a biharmonic map. Non-harmonic biharmonic maps are said to be proper biharmonic maps. In the last decade there has been a growing interest in the theory of biharmonic maps which can be divided in two main research directions. On the one side, the differential geometric aspect has driven attention to the construction of examples and classification results. More precisely, in [3], Caddeo et al. classified biharmonic curves and surfaces of the unit 3 -sphere $\mathbb{S}^{3}$. In fact, they found that they are circles, helices which are geodesics in the Clifford minimal torus and small hyperspheres. The same authors in [4] constructed examples of proper biharmonic submanifolds of $\mathbb{S}^{n}, n>3$. The other side is the analytic aspect from the point view of PDE: biharmonic maps are solutions of a fourth order strongly elliptic semilinear PDE.

We concentrate on the mapping $X:(M, g) \mapsto\left(T M, g_{S}\right)$. It is natural to consider the problem of characterizing those vector fields for which the corresponding map is a biharmonic map. Furthermore, we can also look for vector fields $X$ that are critical points of the bienergy functional restricted to variations among vector fields. In the latter case, such a vector field is called biharmonic vector field. The goal of this paper is to answer to these problems. The paper is organized in the following way. Section 2 contains the presentation of some basic notions about the geometry of the tangent bundle and biharmonic maps. In Section 3, we derive the first variational formula associated to the bienergy functional restricted to the space of all vector fields of $(M, g)$ (see Theorem 3.2). By using this formula, we prove the following Theorem:

Theorem 1.1. Let $(M, g)$ be a compact m-dimensional Riemannian manifold and $X \in \mathfrak{X}(M)$ a tangent vector field. Then, $X$ is a biharmonic vector field if and only if $X$ is parallel. 
As a consequence, we deduce that

Corollary 1.2. Let $(M, g)$ be a compact oriented m-dimensional Riemannian manifold and $X \in \mathfrak{X}(M)$ a tangent vector field. Then, $X$ : $(M, g) \mapsto\left(T M, g_{S}\right)$ is a biharmonic map if and only if $X$ is parallel.

Finally, we give examples of non-parallel biharmonic vector fields of $\left(\mathbb{R}^{2}, g\right)$, equipped with the standard Euclidean metric $g$, which are non harmonic (see Example 3.9 and Remarks 3.10 and 3.11). More precisely, in Remark 3.11, we give the general representation formula of a biharmonic vector field of $\left(\mathbb{R}^{2}, g\right)$. Furthermore, we give examples of biharmonic vector fields which are non harmonic vector fields in the case which the base manifold $(M, g)$ is non-flat (see Examples 3.12 and 3.13). As a consequence, we point out that the notions "biharmonic vector field" and "harmonic vector field" are independent in the sense that it does not exist an immediate relation between them $\left(^{1}\right)$.

\section{Preliminaries.}

2.1. The tangent bundle. We recall some basic facts about the geometry of the tangent bundle. For a more elaborate exposition, we refer to the survey [9]. In the present paper, we denote by $\mathfrak{X}(M)$ the space of all vector fields of a Riemannian manifold $(M, g)$.

Let $(M, g)$ be an $m$-dimensional Riemannian manifold and $\nabla$ the associated Levi-Civita connection. Its Riemann curvature tensor $R$ is defined by

$$
R(X, Y) Z=\nabla_{X} \nabla_{Y} Z-\nabla_{Y} \nabla_{X} Z-\nabla_{[X, Y]} Z
$$

for all vector fields $X, Y$ and $Z$ on $M$. The tangent bundle of a Riemannian manifold $(M, g)$ is denoted by $T M$ and consists of pairs $(x, u)$ where $x$ is a point in $M$ and $u$ a tangent vector to $M$ at $x$. The mapping $\pi: T M \mapsto M:(x, u) \mapsto x$ is the natural projection from $T M$ onto $M$. The tangent space $T_{(x, u)} T M$ at a point $(x, u)$ in $T M$ is a direct sum of the vertical subspace $\mathcal{V}_{(x, u)}=\operatorname{Ker}\left(\left.d \pi\right|_{(x, u)}\right)$ and the horizontal subspace $\mathcal{H}_{(x, u)}$, with respect to the Levi-Civita connection $\nabla$ of $M$ :

$$
T_{(x, u)} T M=\mathcal{H}_{(x, u)} \oplus \mathcal{V}_{(x, u)} .
$$

\footnotetext{
${ }^{1}$ In [5], the authors proved Corollary 1.2 following a quite different approach from that of the paper. We should point out that their paper includes some serious mistakes. More precisely, on page 470 and line 14 , the term $\nabla_{e_{i}}^{X}\left(\tau^{v}(X)\right)^{V}$ should be calculated at the point $\left(x, X_{x}\right) \in T M$ in order to be a section of the pull - back bundle $X^{-1}(T T M)$. As a consequence, this mistake is transferred to the calculation of the term $\operatorname{Tr}_{g} \nabla^{2}\left(\tau^{v}(X)\right)_{(x, u)}^{V}$ (page 470 and line 18)
} 
For any vector $w \in T_{x} M$, there exists a unique vector $w^{h} \in \mathcal{H}_{(x, u)}$ at the point $(x, u) \in T M$, which is called the horizontal lift of $w$ to $(x, u)$, such that $d \pi\left(w^{h}\right)=w$ and a unique vector $w^{v} \in \mathcal{V}_{(x, u)}$, which is called the vertical lift of $w$ to $(x, u)$, such that $w^{v}(d f)=w(f)$ for all functions $f$ on $M$. Hence, every tangent vector $\bar{w} \in T_{(x, u)} T M$ can be decomposed as $\bar{w}=w_{1}^{h}+w_{2}^{v}$ for uniquely determined vectors $w_{1}, w_{2} \in T_{x} M$. The horizontal (respectively, vertical) lift of a vector field $X$ on $M$ to $T M$ is the vector field $X^{h}$ (respectively, $X^{v}$ ) on $T M$ whose value at the point $(x, u)$ is the horizontal (respectively, vertical) lift of $X_{x}$ to $(x, u)$.

The tangent bundle $T M$ of a Riemannian manifold $(M, g)$ can be endowed in a natural way with a Riemannian metric $g_{S}$, the Sasaki metric, depending only on the Riemannian structure $g$ of the base manifold $M$. It is uniquely determined by

$$
g_{S}\left(X^{h}, Y^{h}\right)=g_{S}\left(X^{v}, Y^{v}\right)=g(X, Y) \circ \pi, \quad g_{S}\left(X^{h}, Y^{v}\right)=0
$$

for all vector fields $X$ and $Y$ on $M$. More intuitively, the metric $g_{S}$ is constructed in such a way that the vertical and horizontal subbundles are orthogonal and the bundle map $\pi:\left(T M, g_{S}\right) \mapsto(M, g)$ is a Riemannian submersion.

2.2. Biharmonic maps. Let $(M, g),(N, h)$ be Riemannian manifolds of dimensions $m$ and $n$, respectively, and let $\varphi:(M, g) \mapsto(N, h)$ be a smooth map between them. We denote by $\nabla^{\varphi}$ the connection of the vector bundle $\varphi^{-1} T N$ induced from the Levi-Civita connection $\bar{\nabla}$ of $(N, h)$ and $\nabla$ the Levi-Civita connection of $(M, g)$. Let $D$ be a compact domain of $M$. The energy (integral) of $\varphi$ over $D$ is defined by:

$$
E_{1}(\varphi)=\frac{1}{2} \int_{D}\|d \varphi\|^{2} v_{g}
$$

where $v_{g}$ is the volume element of $(M, g)$.

A smooth map $\varphi:(M, g) \mapsto(N, h)$ is said to be harmonic if it is a critical point of the energy functional for any compact domain $D$. It is well known $([2])$ that the smooth map $\varphi:(M, g) \mapsto(N, h)$ is harmonic if and only if

$$
\tau_{1}(\varphi)=\operatorname{Tr}(\nabla d \varphi)=\sum_{i=1}^{m}\left\{\nabla_{e_{i}}^{\varphi} d \varphi\left(e_{i}\right)-d \varphi\left(\nabla_{e_{i}} e_{i}\right)\right\}=0,
$$

where $\left\{e_{i}\right\}_{i=1}^{m}$ is a local orthonormal frame field of $(M, g)$. The equation $\tau_{1}(\varphi)=0$ is called the harmonic equation.

J. Eells and J. H. Sampson [7] introduced the notion of polyharmonic maps. In this paper, we only consider polyharmonic maps of order two. Such maps are frequently called biharmonic maps. 
More precisely, a smooth map $\varphi:(M, g) \mapsto(N, h)$ is said to be biharmonic if it is a critical point of the bienergy functional:

$$
E_{2}(\varphi)=\frac{1}{2} \int_{D}\left\|\tau_{1}(\varphi)\right\|^{2} v_{g}
$$

over every compact region $D$ of $M$. The corresponding Euler-Lagrange equation associated to the bienergy functional becomes more complicated and it involves the curvature of the target manifold $(N, h)([12])$. We should mention that an harmonic map is automatically a biharmonic map, in fact a minimum of the bienergy functional. We refer to the survey [13] for more information on results related to the theory of biharmonic maps.

A vector field $X$ on $(M, g)$ can be regarded as the immersion $X$ : $(M, g) \mapsto\left(T M, g_{S}\right): x \mapsto\left(x, X_{x}\right) \in T M$ into its tangent bundle $T M$ equipped with the Sasaki metric $g_{S}$. The tension field $\tau_{1}(X)$ is given by $([8])$

$$
\tau_{1}(X)=\left(-\sum_{i=1}^{m} R\left(\nabla_{e_{i}} X, X\right) e_{i}\right)^{h}+\left(\operatorname{Tr} \nabla^{2} X\right)^{v},
$$

where $\left\{e_{i}\right\}_{i=1}^{m}$ is a local orthonormal frame field of $(M, g)$. We mention that the horizontal part of $\tau_{1}(X)$ is appeared with different sign from the same expression in ([8]) due to the different sign of the Riemann curvature tensor. The term $-\operatorname{Tr} \nabla^{2} X$ is equal to the rough Laplacian $\bar{\Delta} X$, that is

$$
\bar{\Delta} X=-\operatorname{Tr} \nabla^{2} X=\sum_{i=1}^{m}\left\{\nabla_{\nabla_{e_{i}} e_{i}} X-\nabla_{e_{i}} \nabla_{e_{i}} X\right\} .
$$

For the sake of convenience, we set $S(X)=\sum_{i=1}^{m} R\left(\nabla_{e_{i}} X, X\right) e_{i}$. It is worth noting that the term $S(X)$ is well-defined independently of the orthonormal frame used. Furthermore, it holds

$$
S(f X)=f^{2} S(X),
$$

for any smooth function $f$ and vector field $X$ of $M$. As a consequence, relation $(2.2)$ is transformed to

$$
\tau_{1}(X)=(-S(X))^{h}+(-\bar{\Delta} X)^{v} .
$$

In the following, we will use the following Lemmas:

Lemma 2.1. Let $(M, g)$ be an m-dimensional Riemannian manifold and $X, Y$ vector fields on $M$. Then the following equation is satisfied:

$$
\Delta(g(X, Y))=g(\bar{\Delta} X, Y)-g(X, \bar{\Delta} Y)-2 \operatorname{div} \theta_{X Y},
$$

where $\theta_{X Y}$ is the 1 -form defined by $\theta_{X Y}(Z)=g\left(X, \nabla_{Z} Y\right),(Z \in \mathfrak{X}(M))$ and $\Delta$ is the ordinary Laplace-Beltrami operator acting on functions. 
Proof. Let $\left\{e_{i}: 1 \leq i \leq m\right\}$ be a local orthonormal frame field of $(M, g)$. Then (due to the definition of the rough Laplacian and $\nabla g=0$ )

$$
\begin{aligned}
\Delta(g(X, Y))= & \sum_{i=1}^{m}\left\{\nabla_{e_{i}} e_{i}(g(X, Y))-e_{i} e_{i}(g(X, Y))\right\} \\
= & \sum_{i=1}^{m}\left\{g\left(\nabla_{\nabla_{e_{i}} e_{i}} X, Y\right)-g\left(\nabla_{e_{i}} \nabla_{e_{i}} X, Y\right)+g\left(X, \nabla_{\nabla_{e_{i}} e_{i}} Y\right)-\right. \\
& \left.-g\left(X, \nabla_{e_{i}} \nabla_{e_{i}} Y\right)-2 g\left(\nabla_{e_{i}} X, \nabla_{e_{i}} Y\right)\right\}=g(\bar{\Delta} X, Y)+ \\
& +g(X, \bar{\Delta} Y)-2 \sum_{i=1}^{m} g\left(\nabla_{e_{i}} X, \nabla_{e_{i}} Y\right)=g(\bar{\Delta} X, Y)+ \\
& +g(X, \bar{\Delta} Y)-2 \sum_{i=1}^{m}\left\{e_{i}\left(g\left(X, \nabla_{e_{i}} Y\right)\right)-g\left(X, \nabla_{\nabla_{e_{i}} e_{i}} Y\right)+\right. \\
& \left.+g\left(X, \nabla_{\nabla_{e_{i}} e_{i}} Y\right)-g\left(X, \nabla_{e_{i}} \nabla_{e_{i}} Y\right)\right\}=g(\bar{\Delta} X, Y)+ \\
& +g(X, \bar{\Delta} Y)-2 \operatorname{div} \theta_{X Y}-2 g(X, \bar{\Delta} Y)=g(\bar{\Delta} X, Y)- \\
& -g(X, \bar{\Delta} Y)-2 \operatorname{div} \theta_{X Y} .
\end{aligned}
$$

Here we made use of the local expression (with respect to the local orthonormal frame field $\left\{e_{i}: 1 \leq i \leq m\right\}$ of the divergence of differential 1-forms i.e.,

$$
\operatorname{div} \omega=\sum_{i=1}^{m}\left(\nabla_{e_{i}} \omega\right) e_{i}, \quad \omega \in \Omega^{1}(M)
$$

Remark 2.2. Lemma 2.1 could be considered as a generalization of the Lemma 2.15 of [6, p. 53].

Lemma 2.3. [10] Let $(M, g)$ be a Riemannian manifold and $X$ a vector field of $M$. Then the following equation is satisfied:

$$
\bar{\Delta}(f X)=(\Delta f) X+f \bar{\Delta} X-2 \nabla_{\text {gradf }} X,
$$

where $f$ being a smooth function of $M$ and gradf the gradient of $f$.

\section{The BIHARMOniCITY OF VECTOR FIELDS}

In this section, we define the bienergy $E_{2}(X)$ of a vector field $X$ of a Riemannian manifold $(M, g)$. Furthermore, we determine the first variational formula of the bienergy functional $E_{2}$ restricted to the space of all vector fields.

Let $(M, g)$ be a compact $m$-dimensional Riemannian manifold, $\left(T M, g_{S}\right)$ its tangent bundle equipped with the Sasaki metric $g_{S}$ and $X$ a tangent vector field on $M$. The bienergy $E_{2}(X)$ of $X$ is defined to be 
the bienergy of the corresponding map $X:(M, g) \mapsto\left(T M, g_{S}\right)$. More precisely, combining relations (2.1) and (2.4), we get

$$
\left.E_{2}(X)=\frac{1}{2} \int_{M}\left\|\tau_{1}(X)\right\|^{2} v_{g}=\frac{1}{2} \int_{M}[g(S(X), S(X))+g(\bar{\Delta} X, \bar{\Delta} X)]\right]_{3.1)} v_{3}
$$

Now, we give the following definition:

Definition 3.1. Let $(M, g)$ be a Riemannian manifold. A vector field $X \in \mathfrak{X}(M)$ is called biharmonic if the corresponding map $X:(M, g) \mapsto$ $\left(T M, g_{S}\right)$ is a critical point for the bienergy functional $E_{2}$, only considering variations among maps defined by vector fields.

In the sequel, we deduce the critical point condition for $E_{2}: \mathfrak{X}(M) \mapsto$ $\mathbb{R}$. We prove the following Theorem:

Theorem 3.2. Let $(M, g)$ be a compact oriented $m$-dimensional Riemannian manifold, $\left\{e_{i}\right\}_{i=1}^{m}$ a local orthonormal frame field of $(M, g)$, $X$ a tangent vector field on $M$ and $E_{2}: \mathfrak{X}(M) \mapsto[0,+\infty)$ the bienergy functional restricted to the space of all vector fields. Then

$$
\begin{aligned}
\frac{d}{d t} E_{2}\left(X_{t}\right)_{t=0}= & \int_{M}\left\{g \left(\bar{\Delta} \bar{\Delta} X+\sum_{i=1}^{m}\left[\left(\nabla_{e_{i}} R\right)\left(e_{i}, S(X)\right) X+R\left(e_{i}, \nabla_{e_{i}} S(X)\right) X+\right.\right.\right. \\
& \left.\left.\left.+2 R\left(e_{i}, S(X)\right) \nabla_{e_{i}} X\right], V\right)\right\} v_{g}
\end{aligned}
$$

for any smooth 1-parameter variation $U: M \times(-\epsilon, \epsilon) \mapsto T M$ of $X$ through vector fields i.e., $X_{t}(z)=U(z, t) \in T_{z} M$ for any $|t|<\epsilon$ and $z \in M$, or equivalently $X_{t} \in \mathfrak{X}(M)$ for any $|t|<\epsilon$. Also, $V$ is the tangent vector field on $M$ given by

$$
V(z)=\frac{d}{d t} X_{z}(0), \quad z \in M,
$$

where $X_{z}(t)=U(z, t),(z, t) \in M \times(-\epsilon, \epsilon)$.

Proof. Let $X$ be a tangent vector on $M$ and $I=(-\epsilon, \epsilon), \epsilon>0$. For $t \in I$, we denote by $i_{t}: M \mapsto M \times I, p \mapsto(p, t)$ the canonical injection. We consider $C^{\infty}$-variations $U: M \times I \mapsto T M$ of $X$ within $\mathfrak{X}(M)$, i.e. for all $t \in I$ the mappings $X_{t}=U \circ i_{t}$ are in fact vector fields and $X_{0}=X$. We choose $\left\{e_{i}\right\}_{i=1}^{m}$ a local orthonormal frame field of $(M, g)$. We extend $e_{i}$ (resp. $\left.\frac{d}{d x} \in \mathfrak{X}(I)\right)$ to $M \times I$, denoted by $E_{i}$ (resp. $\frac{d}{d t}$ ). Moreover, we have $\left[E_{i}, \frac{d}{d t}\right]=0$. We denote by $D$ the Levi-Civita connection of $M \times I$ and $R^{D}$ the Riemann curvature tensor of $M \times I$. Since $M \times I$ is a Riemannian product, we have (using the second Bianchi identity 
for the last relation)

$$
R^{D}(T M, T I)=0, \quad D_{\frac{d}{d t}} E_{i}=0, \quad D_{E_{i}} \frac{d}{d t}=0, \quad\left(D_{\frac{d}{d t}} R^{D}\right)\left(D_{E_{i}} U, U\right) E_{i}=0
$$

for all $1 \leq i \leq m$. We set $Z=\sum_{i=1}^{m} R^{D}\left(D_{E_{i}} U, U\right) E_{i}$ and $\Omega=$ $\sum_{i=1}^{m}\left[D_{D_{E_{i}} E_{i}} U-D_{E_{i}} D_{E_{i}} U\right]$. We easily observe that $S\left(X_{t}\right)=Z \circ i_{t}$ and $\bar{\Delta} X_{t}=\Omega \circ i_{t}$. In the sequel, we consider the function

$$
\begin{aligned}
E_{2}(t) & =E_{2}\left(X_{t}\right)=\frac{1}{2} \int_{M}\left[g\left(S\left(X_{t}\right), S\left(X_{t}\right)\right)+g\left(\bar{\Delta} X_{t}, \bar{\Delta} X_{t}\right)\right] v_{g} \\
& =\frac{1}{2} \int_{M}[g(Z, Z)+g(\Omega, \Omega)] \circ i_{t} v_{g} .
\end{aligned}
$$

Differentiating the function $E_{2}(t)$ at each $t$, we obtain

$$
\frac{d}{d t} E_{2}\left(X_{t}\right)=\int_{M} g\left(D_{\frac{d}{d t}} Z, Z\right) \circ i_{t} v_{g}+\int_{M} g\left(D_{\frac{d}{d t}} \Omega, \Omega\right) \circ i_{t} v_{g}
$$

Taking into account the symmetries of the Riemann curvature tensor, relations (3.3) and summing over all repeated indices, we have

$$
\begin{aligned}
\int_{M} g\left(D_{\frac{d}{d t}} Z, Z\right) \circ i_{t} v_{g}= & \int_{M} g\left(\left(D_{\frac{d}{d t}} R^{D}\right)\left(D_{E_{i}} U, U\right) E_{i}+R^{D}\left(D_{\frac{d}{d t}} D_{E_{i}} U, U\right) E_{i}+\right. \\
& \left.+R^{D}\left(D_{E_{i}} U, D_{\frac{d}{d t}} U\right) E_{i}, Z\right) \circ i_{t} v_{g} \\
= & \int_{M}\left[g\left(R^{D}\left(D_{E_{i}} D_{\frac{d}{d t}} U+R^{D}\left(\frac{d}{d t}, E_{i}\right) U, U\right) E_{i}, Z\right)+\right. \\
& \left.+g\left(R^{D}\left(E_{i}, Z\right) D_{E_{i}} U, D_{\frac{d}{d t}} U\right)\right] \circ i_{t} v_{g} \\
= & \int_{M}\left[-g\left(R^{D}\left(E_{i}, Z\right) U, D_{E_{i}} D_{\frac{d}{d t}} U\right)+\right. \\
& \left.+g\left(R^{D}\left(E_{i}, Z\right) D_{E_{i}} U, D_{\frac{d}{d t}} U\right)\right] \circ i_{t} v_{g} \\
= & \int_{M}\left\{\left[-E_{i}\left(g\left(R^{D}\left(E_{i}, Z\right) U, D_{\frac{d}{d t}} U\right)\right)+g\left(R^{D}\left(D_{E_{i}} E_{i}, Z\right) U, D_{\frac{d}{d t}} U\right)\right]+\right. \\
& +g\left(\left(D_{E_{i}} R^{D}\right)\left(E_{i}, Z\right) U, D_{\frac{d}{d t}} U\right)+g\left(R^{D}\left(E_{i}, D_{E_{i}} Z\right) U, D_{\frac{d}{d t}} U\right)+ \\
& \left.+2 g\left(R\left(E_{i}, Z\right) D_{E_{i}} U, D_{\frac{d}{d t}} U\right)\right\} \circ i_{t} v_{g} .
\end{aligned}
$$


Applying the divergence Theorem for the 1-form $\eta_{t}(W)=g\left(R\left(W, S\left(X_{t}\right)\right) X_{t}, \nabla_{\frac{d}{d t}} X_{t}\right), t \in$ $I, W \in \mathfrak{X}(M)$, relation (3.5) gives

$$
\begin{aligned}
\int_{M} g\left(D_{\frac{d}{d t}} Z, Z\right) \circ i_{t} v_{g}= & \int_{M} g\left(\left(\nabla_{e_{i}} R\right)\left(e_{i}, S\left(X_{t}\right)\right) X_{t}+g\left(R\left(e_{i}, \nabla_{e_{i}} S\left(X_{t}\right)\right) X_{t}+\right.\right. \\
& +2 g\left(R\left(e_{i}, S\left(X_{t}\right)\right) \nabla_{e_{i}} X_{t}, \nabla_{\frac{d}{d t}} X_{t}\right) v_{g} .
\end{aligned}
$$

Similarly, summing over all repeated indices, we deduce

$$
\begin{aligned}
\int_{M} g\left(D_{\frac{d}{d t}} \Omega, \Omega\right) \circ i_{t} v_{g}= & \int_{M} g\left(D_{\frac{d}{d t}} D_{D_{E_{i}} E_{i}} U-D_{\frac{d}{d t}} D_{E_{i}} D_{E_{i}} U, \Omega\right) \circ i_{t} v_{g} \\
= & \int_{M} g\left(D_{D_{E_{i}} E_{i}} D_{\frac{d}{d t}} U-D_{E_{i}} D_{E_{i}} D_{\frac{d}{d t}} U, \Omega\right) \circ i_{t} v_{g} \\
= & \int_{M}\left\{D_{E_{i}} E_{i}\left[g\left(D_{\frac{d}{d t}} U, \Omega\right)\right]-g\left(D_{\frac{d}{d t}} U, D_{D_{E_{i}} E_{i}} \Omega\right)-\right. \\
& \left.-E_{i}\left[g\left(D_{E_{i}} D_{\frac{d}{d t}} U, \Omega\right)\right]+g\left(D_{E_{i}} D_{\frac{d}{d t}} U, D_{E_{i}} \Omega\right)\right\} \circ i_{t} v_{g} \\
= & \int_{M}\left\{D_{E_{i}} E_{i}\left[g\left(D_{\frac{d}{d t}} U, \Omega\right)\right]-E_{i} E_{i}\left[g\left(D_{\frac{d}{d t}} U, \Omega\right)\right]-g\left(D_{\frac{d}{d t}} U, D_{D_{E_{i}} E_{i}} \Omega\right)\right. \\
& \left.+E_{i}\left[g\left(D_{\frac{d}{d t}} U, D_{E_{i}} \Omega\right)\right]+g\left(D_{E_{i}} D_{\frac{d}{d t}} U, D_{E_{i}} \Omega\right)\right\} \circ i_{t} v_{g} \\
= & \int_{M}\left\{\Delta\left[g\left(D_{\frac{d}{d t}} U, \Omega\right)\right]-g\left(D_{\frac{d}{d t}} U, D_{D_{E_{i}} E_{i}} \Omega\right)+2 E_{i}\left[g\left(D_{\frac{d}{d t}} U, D_{E_{i}} \Omega\right)\right]\right. \\
& \left.-g\left(D_{\frac{d}{d t}} U, D_{E_{i}} D_{E_{i}} \Omega\right)\right\} \circ i_{t} v_{g} \\
= & \int_{M}\left\{\Delta\left[g\left(D_{\frac{d}{d t}} U, \Omega\right)\right]-g\left(D_{\frac{d}{d t}} U, D_{D_{E_{i}} E_{i}} \Omega\right)+2 E_{i}\left[g\left(D_{\frac{d}{d t}} U, D_{E_{i}} \Omega\right)\right]\right. \\
& -2 g\left(D_{\frac{d}{d t}} U, D_{D_{E_{i}} E_{i}} \Omega\right)+2 g\left(D_{\frac{d}{d t}} U, D_{D_{E_{i}} E_{i}} \Omega\right)- \\
& \left.-g\left(D_{\frac{d}{d t}} U, D_{E_{i}} D_{E_{i}} \Omega\right)\right\} \circ i_{t} v_{g}
\end{aligned}
$$

Applying the divergence Theorem for the 1-form $\theta_{t}(\cdot)=g\left(\nabla_{\frac{d}{d t}} X_{t}, \nabla \cdot \bar{\Delta} X_{t}\right), t \in$ $I$, we have

$$
\begin{aligned}
\int_{M} g\left(D_{\frac{d}{d t}} \Omega, \Omega\right) \circ i_{t} v_{g} & =\int_{M} \Delta\left[g\left(\nabla_{\frac{d}{d t}} X_{t}, \bar{\Delta} X_{t}\right)\right] v_{g}+2 \int_{M} \operatorname{div} \theta_{t} v_{g}+\int_{M} g\left(\nabla_{\frac{d}{d t}} X_{t}, \bar{\Delta} \bar{\Delta} X_{t}\right) v_{g} \\
& =\int_{M} g\left(\nabla_{\frac{d}{d t}} X_{t}, \bar{\Delta} \bar{\Delta} X_{t}\right) v_{g}
\end{aligned}
$$

Substituting (3.6) and (3.7) in (3.4), evaluating at $t=0$ and setting $V=\left.\nabla_{\frac{d}{d t}} X_{t}\right|_{t=0}$, we easily obtain (3.2).

Since the vector field $X$ is biharmonic if and only if $\left.\frac{d}{d t} E_{2}\left(X_{t}\right)\right|_{t=0}=0$ for all admissible variations, we get 
Corollary 3.3. A vector field $X$ of an m-dimensional Riemannian manifold $(M, g)$ is biharmonic if and only if

$\bar{\Delta} \bar{\Delta} X+\sum_{i=1}^{m}\left[\left(\nabla_{e_{i}} R\right)\left(e_{i}, S(X)\right) X+R\left(e_{i}, \nabla_{e_{i}} S(X)\right) X+2 R\left(e_{i}, S(X)\right) \nabla_{e_{i}} X\right]=0$,

where $\left\{e_{i}\right\}_{i=1}^{m}$ is a local orthonormal frame field of $(M, g)$.

Remark 3.4. Theorem 3.2 holds if $(M, g)$ is a non-compact Riemannian manifold. Indeed, if $M$ is non-compact, you must take an open subset $W$ in $M$ whose closure is compact, and take an arbitrary $V$ but, the support of $V$, namely, the closure of the set of all points in $M$ at which $V$ is not zero, is contained in $W$. Then, (3.2) holds in the form:

$$
\begin{aligned}
\frac{d}{d t} E_{2}\left(X_{t}\right)_{t=0}= & \int_{W}\left\{g \left(\bar{\Delta} \bar{\Delta} X+\sum_{i=1}^{m}\left[\left(\nabla_{e_{i}} R\right)\left(e_{i}, S(X)\right) X+R\left(e_{i}, \nabla_{e_{i}} S(X)\right) X+\right.\right.\right. \\
& \left.\left.\left.+2 R\left(e_{i}, S(X)\right) \nabla_{e_{i}} X\right], V\right)\right\} v_{g}
\end{aligned}
$$

for such any smooth 1-parameter variation $U$. Then, you get that the term $\sum_{i=1}^{m}\left[\left(\nabla_{e_{i}} R\right)\left(e_{i}, S(X)\right) X+R\left(e_{i}, \nabla_{e_{i}} S(X)\right) X+2 R\left(e_{i}, S(X)\right) \nabla_{e_{i}} X\right]+$ $\bar{\Delta} \bar{\Delta} X=0$ vanishes. Then, we obtain Corollary 3.3 in the case which $(M, g)$ is non-compact.

Remark 3.5. In [8], Gil-Medrano proved that a vector field $X$ in a Riemannian manifold $(M, g)$ is a harmonic vector field (i.e. critical point of the energy functional $E_{1}$, only considering variations among maps defined by vector fields) if and only if

$$
\bar{\Delta} X=0 .
$$

The above condition involves only the connection on the Riemannian manifold $(M, g)$. On the contrary, the corresponding critical point condition which characterizes the biharmonic vector fields involves additionally the Riemann curvature tensor of $(M, g)$.

In the following Theorem, we investigate the condition under of which a vector field $X$ of a Riemannian manifold $(M, g)$ is biharmonic under the assumption that the base manifold $(M, g)$ is compact. In particular, we have

Theorem 3.6. Let $(M, g)$ be a compact oriented $m$-dimensional Riemannian manifold and $X \in \mathfrak{X}(M)$ a tangent vector field. Then, $X$ is a biharmonic vector field if and only if $X$ is parallel. 
Proof. We assume that the vector field $X$ is a biharmonic vector field i.e. critical point of the bienergy functional $E_{2}$ restricted to the space of all vector fields of $(M, g)$. We consider the smooth 1-parameter variation $X_{t}=(1+t) X$ of $X(t \in I=(-\epsilon, \epsilon), \epsilon>0)$. By using relations $(2.5),(3.2)$ and the symmetries of the Riemann curvature tensor, we have

$$
\begin{aligned}
0= & \left.\frac{d}{d t} E_{2}\left(X_{t}\right)\right|_{t=0}=\int_{M} g(\bar{\Delta} \bar{\Delta} X, X) v_{g}+\int_{M} g\left(\sum_{i=1}^{m}\left(\nabla_{e_{i}} R\right)\left(e_{i}, S(X)\right) X, X\right) v_{g}+ \\
& +\int_{M} g\left(\sum_{i=1}^{m} R\left(e_{i}, \nabla_{e_{i}} S(X)\right) X, X\right) v_{g}+2 \int_{M} g\left(\sum_{i=1}^{m} R\left(e_{i}, S(X)\right) \nabla_{e_{i}} X, X\right) v_{g} \\
= & \int_{M} \Delta[g(\bar{\Delta} X, X)] v_{g}+\int_{M} g(\bar{\Delta} X, \bar{\Delta} X) v_{g}+2 \int_{M} \operatorname{div} \theta_{\bar{\Delta} X X} v_{g}+ \\
& +2 \int_{M} g(S(X), S(X)) v_{g}=\int_{M}[g(\bar{\Delta} X, \bar{\Delta} X)+2 g(S(X), S(X))] v_{g},
\end{aligned}
$$

where $\left\{e_{i}\right\}_{i=1}^{m}$ a local orthonormal frame field of $(M, g)$. Furthermore, we have applied the divergence Theorem for the function $g(\bar{\Delta} X, X)$ and the 1 -form $\theta_{\bar{\Delta} X X}$. Since both functions $g(\bar{\Delta} X, \bar{\Delta} X)$ and $g(S(X), S(X))$ are positive, we easily conclude that $\bar{\Delta} X=S(X)=0$ everywhere on $M$. Equivalently, $X:(M, g) \mapsto\left(T M, g_{S}\right)$ is an harmonic map. By a result of Nouhaud ([14]), we get that $X$ is parallel. Conversely, we assume that the vector field $X$ is parallel. In this case, $X:(M, g) \mapsto$ $\left(T M, g_{S}\right)$ is an harmonic map $([14])$ and, hence trivially a biharmonic map. As a consequence, $X$ is a critical point of the bienergy functional $E_{2}$ restricted to the set of all vector fields of $(M, g)$.

Corollary 3.7. Let $(M, g)$ be a compact oriented m-dimensional Riemannian manifold and $X \in \mathfrak{X}(M)$ a tangent vector field. Then, $X$ : $(M, g) \mapsto\left(T M, g_{S}\right)$ is a biharmonic map if and only if $X$ is parallel.

Remark 3.8. In [8], Gil-Medrano proved that if $(M, g)$ is compact, then $X$ is critical point of the energy functional $E_{1}$ restricted to the set of all vector fields of $(M, g)$ (i.e. $X$ is a harmonic vector field) if and only if $X$ is parallel. Theorem 3.6 shows that this result of Gil-Medrano remains invariant in the case which the energy functional is substituted by the bienergy functional. Furthermore, Nouhaud ([14]) proved that if $M$ is compact, then $X:(M, g) \mapsto\left(T M, g_{S}\right)$ is an harmonic map if and only if $X$ is parallel (see also [11]). Corollary 3.7 shows that the result of Nouhaud remains also invariant if $X:(M, g) \mapsto\left(T M, g_{S}\right)$ is biharmonic map. 
In the following, we give examples of non-parallel biharmonic vector fields in the case which the base manifold is non-compact. More precisely, we have

Example 3.9. Let $\left(M=\mathbb{R}^{2}, g\right)$ with the cartesian coordinates $(x, y)$, equipped with the standard Euclidean metric $g$. The general form of a vector field $X$ of $\mathbb{R}^{2}$ is

$$
X=f(x, y) \frac{\partial}{\partial x}+g(x, y) \frac{\partial}{\partial y},
$$

where $f, g$ are smooth functions of $\mathbb{R}^{2}$ and $\frac{\partial}{\partial x}, \frac{\partial}{\partial y}$ are the basic vector fields of $\mathbb{R}^{2}$. We have

$$
\begin{aligned}
\bar{\Delta} X & =-\left(f_{x x}+f_{y y}\right) \frac{\partial}{\partial x}-\left(g_{x x}+g_{y y}\right) \frac{\partial}{\partial y}, \\
\bar{\Delta} \bar{\Delta} X & =-\left(f_{x x x x}+2 f_{x x y y}+f_{y y y y}\right) \frac{\partial}{\partial x}-\left(g_{x x x x}+2 g_{x x y y}+g_{y y y y}\right) \frac{\partial}{\partial y},
\end{aligned}
$$

where $f_{x}=\frac{\partial f}{\partial x}, f_{x x}=\frac{\partial^{2} f}{\partial x^{2}}$ etc. By using (3.8), we deduce that the vector field $X$ is biharmonic if and only if $\bar{\Delta} \bar{\Delta} X=0$ or, equivalently,

$$
\Delta^{2} f=f_{x x x x}+2 f_{x x y y}+f_{\text {yyyy }}=0, \quad \Delta^{2} g=g_{x x x x}+2 g_{x x y y}+g_{y y y y}=0 .
$$

Particular solutions of the system (3.9) are

$$
\begin{aligned}
& f(x, y)=\{(A+C x) \cosh \beta x+(B+D x) \sinh \beta x\}\{a \cos \beta y+b \sin \beta y\}, \\
& g(x, y)=\{(A+C x) \cos \beta x+(B+D x) \sin \beta x\}\{a \cosh \beta y+b \sinh \beta y\},
\end{aligned}
$$

where $A, B, C, D, a, b$ and $\beta$ are arbitrary real constants.

Remark 3.10. The equation $\Delta^{2} f=0$ is called biharmonic equation and the corresponding solutions are called biharmonic functions. The biharmonic equation is encountered in plane problems of elasticity ( $f$ is the Airy stress function). It is also used to describe slow flows of viscous incompressible fluids ( $f$ is the stream function). For more information, we refer to the book [15]. Considering the function $f(x, y)$ of the system (3.9) given in (3.10), we calculate

$$
f_{x x}+f_{y y}=2 \beta\{D \cosh \beta x+C \sinh \beta x\}\{a \cos \beta y+b \sin \beta y\} .
$$

Considering the case $\beta=C=D=a=1$ and $b=0$, we obtain $f_{x x}+$ $f_{y y}=2 e^{x} \cos y \neq 0$ for all $(x, y) \in \mathbb{R}^{2}-\left\{\left(t, 2 \kappa \pi+\frac{\pi}{2}\right) / t \in \mathbb{R}, \kappa \in \mathbb{Z}\right\}$. As a conclusion, for this particular choice of the parameters $\beta, C, D, a$ and $b$, the corresponding solution $f(x, y)$ of (3.9) is a biharmonic function 
but not harmonic function. Similarly, for the solution $g(x, y)$ of the system (3.9) given in (3.10), we have

$$
g_{x x}+g_{y y}=2 \beta\{D \cos \beta x-C \sin \beta x\}\{a \cosh \beta y+b \sinh \beta y\} .
$$

Considering the case $a=b=\beta=D=1$ and $C=0$, we obtain $g_{x x}+g_{y y}=2 e^{y} \cos x \neq 0$ for all $(x, y) \in \mathbb{R}^{2}-\left\{\left(2 \kappa \pi+\frac{\pi}{2}, t\right) / \kappa \in \mathbb{Z}, t \in \mathbb{R}\right\}$. Summarizing, we yield that the vector fields $X$ of $\mathbb{R}^{2}$

$X=\{(A+x) \cosh x+(B+x) \sinh x\} \cos y \frac{\partial}{\partial x}+e^{y}\{A \cos x+(B+x) \sin x\} \frac{\partial}{\partial y}$,

where $A, B$ are arbitrary real constants, are non-parallel biharmonic vector fields but non harmonic (see Remark 3.5).

Remark 3.11. Given $u(x, y)$ and $v(x, y)$ solutions of the Laplace equation $(\Delta w=0)$, we have the following various representations of the general solution of the biharmonic equation $\Delta^{2} w=0$ ([15, p. 516]):

$$
\begin{aligned}
& w(x, y)=x u(x, y)+v(x, y) \\
& w(x, y)=y u(x, y)+v(x, y) \\
& w(x, y)=\left(x^{2}+y^{2}\right) u(x, y)+v(x, y) .
\end{aligned}
$$

Equivalently, we have the following complex form of the presentation of the general solution:

$$
w(x, y)=\operatorname{Re}[\bar{z} f(z)+g(z)]
$$

where $f(z)$ and $g(z)$ are arbitrary analytic functions of the variable $z=x+i y, \bar{z}=x-i y$ (see also [1]). As a consequence, the general representation formula of a biharmonic vector field $X$ of $\left(\mathbb{R}^{2}, g\right)$ is

$$
X=(x u(x, y)+v(x, y)) \frac{\partial}{\partial x}+(y u(x, y)+v(x, y)) \frac{\partial}{\partial y},
$$

where $u(x, y)$ and $v(x, y)$ are arbitrary harmonic functions.

Example 3.12. We consider the Heisenberg group $\mathrm{Nil}_{3}$. This is defined to be the group consisting of all real $3 \times 3$ upper triangular matrices of the form

$$
A=\left(\begin{array}{lll}
1 & x & y \\
0 & 1 & z \\
0 & 0 & 1
\end{array}\right)
$$

endowed with the left-invariant metric given by $(d x)^{2}+(d y-x d z)^{2}+$ $(d z)^{2}$. We may thus identify $\mathrm{Nil}_{3}$ with $\mathbb{R}^{3}$ endowed with this metric. The left-invariant vector fields

$$
e_{1}=\frac{\partial}{\partial x}, \quad e_{2}=\frac{\partial}{\partial y}, \quad e_{3}=\frac{\partial}{\partial z}+x \frac{\partial}{\partial y},
$$


constitute an orthonormal basis of the Lie algebra $\mathfrak{g}$ of $\mathrm{Nil}_{3}$. The corresponding Levi Civita connection is determined by

$$
\nabla_{e_{1}} e_{2}=\nabla_{e_{2}} e_{1}=-\frac{1}{2} e_{3}, \quad \nabla_{e_{1}} e_{3}=-\nabla_{e_{3}} e_{1}=\frac{1}{2} e_{2}, \quad \nabla_{e_{2}} e_{3}=\nabla_{e_{3}} e_{2}=\frac{1}{2} e_{1},
$$

where the remaining covariant derivatives of the basic vectors vanish. By using relations (3.11), we get

$$
\begin{gathered}
R\left(e_{2}, e_{1}\right) e_{3}=R\left(e_{2}, e_{3}\right) e_{1}=R\left(e_{3}, e_{1}\right) e_{2}=0 \\
\bar{\Delta} e_{1}=-\nabla_{e_{1}} \nabla_{e_{1}} e_{1}-\nabla_{e_{2}} \nabla_{e_{2}} e_{1}-\nabla_{e_{3}} \nabla_{e_{3}} e_{1}=\frac{1}{2} e_{1}, \quad \bar{\Delta} e_{2}=\frac{1}{2} e_{2}, \quad \bar{\Delta} e_{3}=\frac{1}{2} e_{3} .
\end{gathered}
$$

We consider the vector field $X=f(x) e_{1}$, where $f(x)$ is a smooth function of $\mathbb{R}$ depending of the variable $x$. Combining relations (2.3), (2.6), (3.11) and (3.12), we obtain

$$
\begin{aligned}
\bar{\Delta} X & =\Delta f e_{1}+f \bar{\Delta} e_{1}-2 f^{\prime} \nabla_{e_{1}} e_{1}=\left(\frac{1}{2} f-f^{\prime \prime}\right) e_{1}, \\
\bar{\Delta} \bar{\Delta} X & =\Delta\left(\frac{1}{2} f-f^{\prime \prime}\right) e_{1}+\left(\frac{1}{2} f-f^{\prime \prime}\right) \bar{\Delta} e_{1}=\left(f^{\prime \prime \prime \prime}-f^{\prime \prime}+\frac{1}{4} f\right) e_{1}, \\
S(X) & =f^{2} S\left(e_{1}\right)=f^{2}\left(-\frac{1}{2} R\left(e_{3}, e_{1}\right) e_{2}-\frac{1}{2} R\left(e_{2}, e_{1}\right) e_{3}\right)=0,
\end{aligned}
$$

where $f^{\prime}=\frac{d f}{d x}, f^{\prime \prime}=\frac{d^{2} f}{d x^{2}}$ etc.By using (3.8), we deduce that the vector field $X$ is biharmonic if and only if $\bar{\Delta} \bar{\Delta} X=0$ or, equivalently,

$$
f^{\prime \prime \prime \prime}-f^{\prime \prime}+\frac{1}{4} f=0 .
$$

The general solution of the homogeneous fourth order equation (3.13) is

$$
f(x)=c_{1} e^{\frac{x}{\sqrt{2}}}+c_{2} x e^{\frac{x}{\sqrt{2}}}+c_{3} e^{-\frac{x}{\sqrt{2}}}+c_{4} x e^{-\frac{x}{\sqrt{2}}},
$$

where $c_{1}, c_{2}, c_{3}$ and $c_{4}$ are real constants. We easily conclude that the vector fields $X=\left[c_{1} e^{\frac{x}{\sqrt{2}}}+c_{3} e^{-\frac{x}{\sqrt{2}}}\right] e_{1}, c_{1}, c_{3} \in \mathbb{R}$ are harmonic and, also, biharmonic vector fields. On the contrary, the vector fields $X=$ $x\left(c_{2} e^{\frac{x}{\sqrt{2}}}+c_{4} e^{-\frac{x}{\sqrt{2}}}\right) e_{1}$ are biharmonic but non harmonic vector fields of $\mathrm{Nil}_{3}$. Following the same procedure, the vector fields $X=z\left(c_{2} e^{\frac{z}{\sqrt{2}}}+\right.$ $\left.c_{4} e^{-\frac{z}{\sqrt{2}}}\right) e_{3}, c_{2}, c_{4} \in \mathbb{R}$ are also biharmonic but non harmonic vector fields of $\mathrm{Nil}_{3}$.

In the following example, we give examples of vector fields which are harmonic vector fields but not biharmonic vector fields. More precisely, we have 
Example 3.13. We consider the hyperbolic space $\left(H^{n}, g\right)$ of constant sectional curvature $-c^{2}<0$

$$
\begin{aligned}
H^{n} & =\left\{z=\left(x_{1}, \ldots, x_{n-1}, y\right) \in \mathbb{R}^{n}: y>0\right\}, \\
g & =\frac{1}{(c y)^{2}}\left(\sum_{i=1}^{n-1}\left(d x_{i}\right)^{2}+(d y)^{2}\right) .
\end{aligned}
$$

The vector fields

$$
V=c y \frac{\partial}{\partial y}, \quad E_{i}=c y \frac{\partial}{\partial x_{i}}, \quad 1 \leq i \leq n-1,
$$

constitute an orthonormal frame field of $\left(H^{n}, g\right)$ and

$$
\left[V, E_{i}\right]=c E_{i}, \quad\left[E_{i}, E_{j}\right]=0, \quad 1 \leq i, j \leq n-1 .
$$

Let $\nabla$ be the Levi-Civita connection of $\left(H^{n}, g\right)$. It follows that ([6, p. 139])

$$
\nabla_{E_{i}} V=-c E_{i}, \quad \nabla_{V} V=0, \quad \nabla_{V} E_{i}=0, \quad \nabla_{E_{i}} E_{j}=c \delta_{i j} V
$$

for any $1 \leq i, j \leq n-1$. Then $([6, \mathrm{p} .139])$

$$
\begin{aligned}
& \bar{\Delta} V=(n-1) c^{2} V, \quad S(V)=-c^{2}\left(\nabla_{V} V-\operatorname{div}(V) V\right)=-c^{3}(n-1) V \\
& \operatorname{div}(V)=-(n-1) c .
\end{aligned}
$$

We consider the vector field $X=f(y) V$, where $f(y)$ is a smooth function of $\mathbb{R}^{+}$depending on the variable $y(y>0)$. By using relations (2.3), (2.6), (3.14) and (3.15), we get

$$
\begin{aligned}
\bar{\Delta} X= & \Delta f V+f \bar{\Delta} V-2 V(f) \nabla_{V} V=[(n-1) c V(f)-V V(f)] V+(n-1) c^{2} f V \\
= & c^{2}\left((n-2) y f^{\prime}-y^{2} f^{\prime \prime}+(n-1) f\right) V \\
\bar{\Delta} \bar{\Delta} X= & c^{2}\left(\Delta\left[(n-2) y f^{\prime}-y^{2} f^{\prime \prime}+(n-1) f\right] V+\left[(n-2) y f^{\prime}-y^{2} f^{\prime \prime}+(n-1) f\right] \bar{\Delta} V\right) \\
= & c^{4}\left(y^{4} f^{\prime \prime \prime \prime}+(8-2 n) y^{3} f^{\prime \prime \prime}+(n-8)(n-2) y^{2} f^{\prime \prime}+(n-2)(3 n-4) y f^{\prime}+\right. \\
& \left.+(n-1)^{2} f\right) V, S(X)=f^{2} S(V)=-c^{3}(n-1) f^{2} V,
\end{aligned}
$$

where $f^{\prime}=\frac{d f}{d y}, f^{\prime \prime}=\frac{d^{2} f}{d y^{2}}$ etc. By using relations (3.14), (3.16) and the fact that $\left(H^{n}, g\right)$ is a space of constant curvature $-c^{2}$, we have

$$
\nabla_{E_{i}} X=-c f E_{i}, \quad \nabla_{V} S(X)=-c^{3}(n-1) V\left(f^{2}\right) V, \quad \nabla_{E_{i}} S(X)=c^{4}(n-1) f^{2} E_{i},
$$




$$
\begin{gathered}
R\left(V, \nabla_{V} S(X)\right) V=0, \quad R\left(E_{i}, \nabla_{E_{i}} S(X)\right) X=0, \quad R(V, S(X)) \nabla_{V} X=0, \\
\sum_{i=1}^{n-1} R\left(E_{i}, S(X)\right) \nabla_{E_{i}} X=c^{6}(n-1)^{2} f^{3} V, \quad \nabla R=0 .
\end{gathered}
$$

for any $1 \leq i \leq n-1$. By using the first relation of (3.16), we easily conclude that the vector field $X=f(y) V$ is a harmonic vector field (equivalently, $\bar{\Delta} X=0$ ) if and only if

$$
y^{2} f^{\prime \prime}-(n-2) y f^{\prime}-(n-1) f=0 .
$$

The ODE (3.18) is a second order Euler's equation and its general solution is

$$
f(y)=c_{1} y^{\frac{n-1-\sqrt{(n-1)(n+3)}}{2}}+c_{2} y^{\frac{n-1+\sqrt{(n-1)(n+3)}}{2}}, y>0
$$

where $c_{1}$ and $c_{2}$ are real constants. On the other hand, combining relations (3.8), (3.16) and (3.17), we easily conclude that the vector field $X=f(y) V$ is biharmonic if and only if

$$
\begin{array}{r}
y^{4} f^{\prime \prime \prime \prime}+(8-2 n) y^{3} f^{\prime \prime \prime}+(n-8)(n-2) y^{2} f^{\prime \prime}+(n-2)(3 n-4) y f^{\prime}+ \\
+(n-1)^{2} f=-2 c^{2}(n-1)^{2} f^{3} .
\end{array}
$$

Clearly, the vector fields $X=y^{\frac{n-1-\sqrt{(n-1)(n+3)}}{2}} V$ and $X=y^{\frac{n-1+\sqrt{(n-1)(n+3)}}{2}} V$ are harmonic vector fields but not biharmonic vector fields.

Remark 3.14. The fourth order ODE (3.19) has a particular interest. Applying the substitution $y=e^{t}$, equation (3.19) is transformed to

$$
\begin{array}{r}
v^{\prime \prime \prime \prime}+(2-2 n) v^{\prime \prime \prime}+(n-1)(n-3) v^{\prime \prime}+2(n-1)^{2} v^{\prime}+(n-1)^{2} v+ \\
+2 c^{2}(n-1)^{2} v^{3}=0,
\end{array}
$$

where $v(t)=f(y)$ and $t \in \mathbb{R}$. The ODE (3.20) has no global solution, since the term $v^{3}$ goes up very quickly at infinity. However, the ODE (3.20) has a local solution.

Remark 3.15. Examples 3.9, 3.12 and 3.13 show that there exist biharmonic vector fields which are not harmonic, and conversely. As a consequence, the notions "biharmonic vector field" and "harmonic vector field" are independent in the sense that it does not exist an immediate relation between them.

Acknowledgment. The authors acknowledge Professor Hisashi Naito for his suggestion about the non existence of global solution of the ODE (3.19). 


\section{REFERENCES}

[1] N. Aronszajn, T. M. Creese and L. J. Lipkin, Polyharmonic functions, Oxford, 1983.

[2] P. Baird and J. C. Wood, Harmonic morphisms between Riemannian manifolds, Oxford University Press, 29, London Math. Soc. Monogr. (N.S.), 2003.

[3] R. Caddeo, S. Montaldo, and C. Oniciuc, Biharmonic submanifolds of $\mathbb{S}^{3}$, Intern. J. Math, 12 (2001), 867-876.

[4] R. Caddeo, S. Montaldo, and C. Oniciuc, Biharmonic submanifolds in spheres, Israel J.Math., 130 (2002), 109-123.

[5] Mustapha Djaa, Hichem Elhendi and Seddik Ouakkas, On the biharmonic vector fields, Turk. J. Math., 36 (2012), 463-474.

[6] S. Dragomir and D. Perrone, Harmonic Vector Fields: Variational Principles and Differential Geometry, Elsevier 2011.

[7] J. Eells and J. H. Sampson, Harmonic mappings of Riemannian manifolds, Amer. J. Math., 86(1) (1964), 109-160.

[8] O. Gil-Medrano, Relationship between volume and energy of unit vector fields, Differential Geom. Appl., 15 (2001), 137-152.

[9] S. Gudmundsson and E. Kappos, On the Geometry of Tangent Bundles, Expo. Math., 20 (2002), 1-41.

[10] A. Higuchi, B. S. Kay and C. M. Wood, The energy of unit vector fields on the 3-sphere, J.Geom.Phys., 37(1-2) (2001), 137-155.

[11] T. Ishihara, Harmonic sections of tangent bundles, J. Math. Tokushima Univ., 13 (1979), 23-27.

[12] G. Jiang, 2-harmonic maps and their first and second variational formulas, Translated into English by Hajime Urakawa, Note Mat., 28 suppl. n. 1 (2008), 209-232.

[13] S. Montaldo and C. Oniciuc, A short survey on biharmonic maps between Riemannian manifolds, Rev. Un. Mat. Argentina, 47(2) (2006), 1-22.

[14] O. Nouhaud, Applications harmoniques d' une variété Riemannienne dans son fibré tangent, C.R.Acad.Sci.Paris, 284 (1977), 815-818.

[15] A. D. Polyanin, Handbook of Linear Partial Differential Equations for Engineers and Scientists, Chapman and Hall/CRC, 2002.

[16] H. Urakawa, Calculus of Variations and Harmonic Maps, Amer. Math. Soc., Providence 132, Transl. Math. Monograph, 1993.

Pafsania, Isthmia Korinthias,

Korinthos, GR-20010, Greece.

E-mail address: mmarkellos@hotmail.gr

Institute for International Education, Tohoku University, Kawauchi 41, Sendai, 980-8576, Japan. E-mail address: urakawa@math.is.tohoku.ac.jp 